In Cres. Vol. $4 N^{\circ} 1:$ pp. 41-49, 2013

\title{
UNA PROPUESTA DIDÁCTICA PARA LA ENSEÑANZA DE LOS CUADRILÁTEROS BASADA EN EL MODELO VAN HIELE*
}

\author{
A METHODOLOGICAL APPROACH TO THE TEACHING \\ OF QUADRILATERALS BASED IN \\ VAN HIELE MODEL
}

Albert Thomy Maguiña Rojas ${ }^{1}$

\section{RESUMEN}

Este trabajo tuvo por objetivo diseñar una propuesta didáctica basada en las fases de aprendizaje del Modelo Van Hiele, para facilitar el ascenso de los estudiantes de un nivel de razonamiento al inmediato superior. Ello permitió analizar y comparar los grados de adquisición que tenía un grupo de alumnos en torno del objeto matemático cuadriláteros, antes y después de implementar la mencionada propuesta. Participaron 10 alumnos del cuarto año de educación secundaria de la institución educativa particular Buenas Nuevas de la ciudad de Lima. Se logró que los alumnos incrementaran los grados de adquisición en los niveles de visualización, análisis y deducción informal, de acuerdo al Modelo Van Hiele.

PALABRAS CLAVE: Modelo Van Hiele, cuadriláteros, didáctica de la geometría.

* Recibido: 20 de marzo del 2013; aprobado: 23 de abril del 2013.

1 Licenciado en Educación con mención Matemática, Física y Computación. Egresado de Postgrado. Pontificia Universidad Católica del Perú. Programa: Magister en la Enseñanza de las Matemáticas. 


\begin{abstract}
This work was aimed to design a didactic based learning phases Van Hiele Model, in order to facilitate the promotion of students from one level to the next higher reasoning. This made it possible to analyze and compare the degrees of acquisition that had a group of students around quadrilaterals mathematical object before and after implementing the above proposal. In the experiment involved 10 students of the fourth year of secondary education particularly school Buenas Nuevas of Lima. The methodology we used for this work, is based on the doctoral thesis "Contributions to the implementation and application of Van Hiele Model" presented by Jaime (1993). Through this teaching proposal that pupils achieved grades acquisition increase in the levels of visualization, analysis and deduction informal, according to Van Hiele Model.
\end{abstract}

KEY WORDS: Model Van Hiele, quadrilaterals, teaching geometry.

\title{
I. INTRODUCCIÓN
}

La geometría es considerada como uno de los pilares fundamentales para el desarrollo del razonamiento lógico. Sin embargo, durante el siglo pasado fue relegada paulatinamente de los planes de estudio. Afortunadamente, esta tendencia está cambiando, devolviendo a esta disciplina su rol como actor principal, tanto en el aprendizaje de las matemáticas como en el desarrollo del pensamiento lógico. Este cambio se evidencia en el Diseño Curricular Nacional (DCN) ${ }^{1}$, elaborado por el Ministerio de Educación del Perú (MINEDU), el cual articula el contenido a lo largo de los tres niveles de estudio, inicial, primaria y secundaria, al considerarlo como uno de sus ejes fundamentales (número y relaciones, geometría y medida, estadística y probabilidades).

No obstante, y a pesar de todos los esfuerzos realizados por el MINEDU, los resultados de los estudiantes en las evaluaciones nacionales, lamentablemente, no son nada alentadoras. En la tabla 01 se muestran los resultados del informe pedagógico elaborado por el MINEDU en el 2001.

Tabla 01

PORCENTAJE DE ESTUDIANTES SEGÚN NIVELES DE DESEMPEÑO

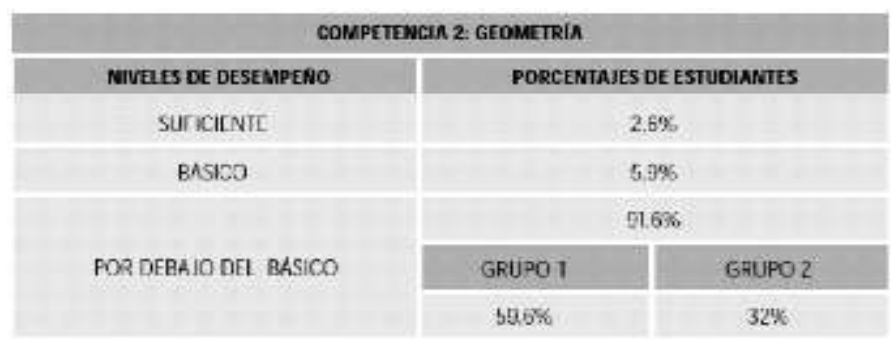

Fuente: Unidad de Medición de la Calidad Educativa (2001, p. 40). 
La experiencia docente indica que los dos temas esenciales para el aprendizaje de la geometría son los triángulos y los cuadriláteros. El último tema fue escogido como objeto matemático de esta investigación, por la preponderancia que ejerce sobre los demás tópicos de la geometría. Al abordar este objeto matemático, se revisó las nociones de paralelismo y de perpendicularidad (rectángulo, rombo, etc.), la noción de punto medio (teorema de la base media, la diagonales se bisecan, etc.); es decir, se desarrolló un conjunto de nociones y propiedades, de los cuadriláteros, que son fundamentales para temas posteriores.

Desde esta perspectiva, diversas investigaciones han centrado su interés en la identificación y análisis de las dificultades asociadas a la enseñanza y aprendizaje de este objeto matemático.

En este sentido, Renzulli y Scaglia ${ }^{2}$, en su investigación sobre clasificación de cuadriláteros, han encontrado fenómenos asociados en los estudiantes, como la formación de esquemas mentales (prototipos) de las figuras geométricas que están fuertemente marcados por características irrelevantes desde el punto de vista conceptual. Afirman que estos prototipos se forman, entre otras razones, por el uso, casi exclusivo, de representaciones gráficas estereotipadas durante la enseñanza de los objetos geométricos.

En este trabajo se abordó el tema de los cuadriláteros a partir de una secuencia didáctica que contribuya al desarrollo del pensamiento geométrico. Para ello, el Modelo de Van Hiele proporcionó las herramientas necesarias para observar el desarrollo del pensamiento geométrico de los estudiantes del cuarto grado de secundaria. A su vez, se usó el software GeoGebra, el cual facilitó la visualización y manipulación del objeto matemático.

\section{PROBLEMA}

¿Qué nivel de pensamiento geométrico, de acuerdo al Modelo Van Hiele, pueden alcanzar los estudiantes del cuarto grado de secundaria en el estudio de los cuadriláteros, utilizando el software GeoGebra?

\section{OBJETIVO GENERAL}

Diseñar una propuesta didáctica, según el Modelo Van Hiele, para promover que los estudiantes del cuarto grado de secundaria alcancen el nivel 3, de deducción informal, haciendo uso del software de geometría dinámica GeoGebra. 


\section{OBJETIVOS ESPECÍFICOS}

1. Elaborar una prueba de entrada como diagnóstico para reconocer el nivel de razonamiento geométrico de los estudiantes de cuarto año de educación secundaria.

2. Diseñar actividades para facilitar la comprensión de los cuadriláteros y mejorar los grados de adquisición en los niveles de visualización, análisis y deducción informal, teniendo en cuenta las fases del modelo Van Hiele y haciendo uso del software GeoGebra de geometría dinámica.

3. Aplicar las prueba de entrada, la prueba de salida y las actividades a los estudiantes de cuarto año de educación secundaria.

4. Analizar los resultados de estas actividades a partir de los niveles de pensamiento geométrico del modelo Van Hiele.

5. Analizar y comparar los resultados de las pruebas de entrada y de salida.

\section{BASES TEÓRICAS}

El modelo de los esposos Van Hiele ${ }^{3}$ tiene su origen en 1957, en las disertaciones doctorales de Dina Van Hiele-Geldof y Pierre Van Hiele en la Universidad de Utirecht, Holanda. El libro original donde se desarrolla la teoría es Structure and Insight: A theory of mathematics education (Estructura y Reflexiones: Una teoría de la educación matemática).

Este modelo ha sido utilizado en diversas ocasiones como base para la elaboración de cursos de geometría e incluso en currículos nacionales de matemática de enseñanza elemental, caso de Holanda y la ex Unión de Repúblicas Socialistas Soviéticas.

En la base del aprendizaje de la geometría hay dos elementos importantes: el lenguaje utilizado y la significatividad de los contenidos. El primero implica que los niveles y su adquisición van unidos al lenguaje adecuado y, el segundo, que sólo se asimila aquello que se presente en el nivel de razonamiento que le corresponde a la persona; si no es así, se debe esperar a que lo alcance para enseñar un nuevo contenido matemático.

Los componentes principales del modelo Van Hiele son la "teoría de los niveles de razonamiento", que explica cómo se produce el desarrollo en la calidad de razonamiento geométrico de los estudiantes, cuando se estudia geometría, y las "fases de aprendizaje", que constituye su propuesta didáctica para la secuenciación de actividades de enseñanza-aprendizaje en el aula, con el objeto de facilitar el ascenso de los estudiantes de un nivel de razonamiento al inmediato superior. 


\section{LOS NIVELES DE RAZONAMIENTO}

La descripción que aquí se expone está tomada de la que utilizaron Shaughnessy y Burger en 1985 (citado por Corberán et al., 1989, p. 14) ${ }^{4}$.

\section{NIVEL 1: VisUALIZACIÓN}

Una figura geométrica es vista como un todo desprovisto de componentes o atributos. Las descripciones reflejan experiencias puramente visuales, hasta el punto de que un alumno de este nivel, a la pregunta de por qué una figura es un rectángulo, responde diciendo que porque se parece, por ejemplo, a una puerta o a una ventana. Un alumno en este nivel puede aprender vocabulario geométrico, puede identificar formas geométricas determinadas de entre un conjunto de ellas y, dada una figura, puede reproducirla.

\section{NIVEL 2: ANÁLISIS}

El alumno analiza de un modo informal las propiedades de las figuras percibidas mediante procesos de observación y experimentación. Empieza a establecerse las propiedades esenciales de los conceptos, aunque todavía es incapaz de ver relaciones entre propiedades y entre figuras. Tampoco es capaz de elaborar o entender definiciones. En este nivel, un alumno diría, por ejemplo, que "un rectángulo es una figura geométrica con cuatro lados, cuatro ángulos, lados opuestos paralelos, ángulos iguales,...”; es decir, aportaría toda una retahíla de propiedades.

\section{NiVEL 3: DEDUCCIÓN INFORMAL}

El alumno ordena lógicamente las propiedades de los conceptos, empieza a construir definiciones abstractas y puede distinguir entre necesidad y suficiencia de un conjunto de propiedades en la determinación de un concepto. En este nivel, puede seguir y dar argumentos informales, pero no comprende el significado de la deducción o el papel de los axiomas. Puede seguir demostraciones formales, pero no puede construir una demostración partiendo de premisas diferentes.

\section{Nivel 4: DEDUCCIÓN FORMAL}

El alumno razona formalmente dentro del contexto de un sistema matemático con términos indefinidos, axiomas, un sistema lógico subyacente, definiciones y teoremas. En este nivel, un alumno es capaz de construir, ya no memorizar, demostraciones. Se puede estudiar la posibilidad de que una demostración se desarrolle siguiendo más de una secuencia de proposiciones. Se entiende la interacción entre condición necesaria y suficiente. 


\section{NIVEL 5: RIGOR}

El alumno puede comparar sistemas basados en axiomáticas diferentes y puede estudiar distintas geometrías en ausencia de modelos concretos. Este nivel es prácticamente inalcanzable por un estudiante de secundaria; por ello, la mayoría de los trabajos de investigación se centran en los tres primeros. El propio Pierre Marie Van Hiele reconoce su interés casi exclusivo por los tres primeros niveles.

De acuerdo a lo planteado por el propio Van Hiele, y teniendo en cuenta los escasos saberes previos de nuestros estudiantes respecto al tema de los cuadriláteros, es idóneo lo propuesto en nuestro objetivo general.

\section{LAS FASES DE APRENDIZAJE}

\section{FASE 1: ENCUESTA/ INFORMACIÓN}

El profesor determina, mediante el diálogo con los estudiantes, dos aspectos importantes: 1) El conocimiento previo sobre el concepto que se va a tratar; y 2) La dirección que tomará el estudio con posterioridad y toda observación que sea pertinente. En esta fase se introduce el vocabulario específico del nivel que se trate.

\section{FASE 2: ORIENTACIÓN DIRIGIDA}

Sobre el concepto a estudiar, los estudiantes exploran dicho concepto a través de los materiales que de forma secuencializada les presenta el profesor, de tal manera que las actividades progresivas revelen las estructuras características de cada nivel. Las cuestiones a plantear por el profesor deben ser concisas $\mathrm{y}$ sin ambigüedad.

\section{FASE 3: EXPLICITACIÓN}

Partiendo de sus experiencias previas, los estudiantes expresan e intercambian sus opiniones acerca de las estructuras observadas. En esta fase, se explicita el sistema de relaciones exploradas. El papel del profesor es mínimo, si bien debe cuidar que el lenguaje del alumno sea el apropiado a su nivel.

\section{FASE 4: ORIENTACIÓN LIBRE}

El alumno se enfrenta a tareas más complejas, trabajos con muchas etapas y que pueden concluirse por distintos procedimientos. El objetivo de esta fase es la consolidación de los conocimientos adquiridos y su aplicación a situaciones inéditas, aunque de estructura comparable a las estudiadas previamente. 


\section{FASE 5: INTEGRACIÓN}

El estudiante revisa, sumariza y unifica los objetos y sus relaciones que configuran el nuevo sistema de conocimientos. En esta fase, no se presenta nada nuevo; simplemente se plantea como síntesis de lo ya hecho y, en todo caso, la revisión de los orígenes de esa síntesis.

\section{METODOLOGÍA}

Para la evaluación de un nivel de razonamiento se considera que este tiene un grado de adquisición, el cual permite observar el mayor o menor dominio de un determinado nivel de razonamiento. Este dominio va desde un dominio nulo (inicio del proceso) hasta un dominio completo (final del proceso). A cada uno de estos dominios, se le ha asignado un nivel, que Jaime (1993 p. 265-266) caracteriza de la siguiente manera:

1. Adquisición nula: no se emplean las características de este nivel de razonamiento.

2. Adquisición baja: empieza la consciencia de las características, métodos y exigencias propios del nivel, pero la utilización que se hace de ellas es muy pobre. Es frecuente que el estudiante abandone el nivel para trabajar en el nivel anterior.

3. Adquisición intermedia: el empleo de los métodos de este nivel es más frecuente y preciso; sin embargo, ante la aparición de alguna dificultad y considerando que el dominio no es completo, se realiza un retroceso al nivel anterior intentando regresar al actual, nuevamente. Por lo tanto, en este proceso hay saltos entre dos niveles consecutivos de razonamiento.

4. Adquisición alta: se tiene como nivel de trabajo habitual el actual, aunque de vez en cuando se produce el retroceso al nivel anterior. En algunas ocasiones, se hace uso inadecuado de las herramientas propias del nivel de razonamiento.

5. Adquisición completa: hay dominio total de las herramientas y métodos de trabajos, propios de este nivel de razonamiento.

En su propuesta, Jaime indica que los porcentajes y la cantidad de divisiones son subjetivos, dado que estas han sido propuestas a partir de experimentaciones realizadas en investigaciones anteriores.

Jaime y Gutiérrez hacen una diferenciación de los tipos de respuestas que los estudiantes pueden presentar. Para ello, definen unos tipos de respuestas, enmarcados dentro de los parámetros del nivel de razonamiento que se está analizando. 
Los tipos de respuestas, según Jaime y Gutiérrez son:

Tipo 1: ítems sin respuesta, con respuesta no codificable o con respuestas que indican que el estudiante no está en un determinado nivel de razonamiento y que no proporcionan ninguna información sobre la forma de utilizar los niveles de razonamiento inferiores.

Tipo 2: respuestas matemáticamente incorrectas e incompletas, pero en las que se reconocen indicios de utilización de cierto nivel de razonamiento.

Tipo 3: respuestas matemáticamente correctas, pero incompletas, en las que se reconocen indicios de utilización de cierto nivel de razonamiento.

Tipo 4: respuestas que reflejan claramente la utilización de dos niveles de razonamiento consecutivos

Tipo 5: respuestas completas, pero matemáticamente incorrectas, que muestran claramente la utilización de determinado nivel de razonamiento.

Tipo 6: respuestas completas y matemáticamente correctas que muestran claramente la utilización predominante de un nivel de razonamiento determinado, pero que aún presentan errores.

Tipo 7: respuestas completas y matemáticamente correctas, que muestran la utilización de un nivel de razonamiento determinado.

\section{COEFICIENTE DE GUTTMAN}

El coeficiente de Guttman validó, de alguna manera, los ítems utilizados en las pruebas, con relación a los niveles. Para el cálculo de estos coeficientes se consideraron los vectores formados por los grados de adquisición de los tres niveles considerados en la prueba $\left(\mathrm{g}_{1}, \mathrm{~g}_{2}, \mathrm{~g}_{3}\right)$.

La situación ideal es $g_{1}>g_{2}>g_{3}$. Se considera que hay un error cuando $g_{x}$ $<\mathrm{g}_{\mathrm{y}}$ siendo $\mathrm{y}>\mathrm{x}$.

Cuando no hay error, se debe obtener un valor de $G=1$, y se considera como límite inferior para aceptar la jerarquización $\mathrm{G}=0,90$ (Mayberry, 1983) ${ }^{6}$

\section{CONCLUSIONES}

A través de la prueba de entrada se logró establecer los saberes previos que poseían los estudiantes antes de implementar la propuesta didáctica, basada en los niveles y fases del modelo Van Hiele.

La propuesta didáctica permitió que los estudiantes lograran un grado de adquisición alta en el nivel 1, un grado de adquisición intermedia en el nivel 2 y 
se encuentren desarrollando habilidades en el nivel 3, de pasar de un nivel de adquisición nula a una baja en este nivel.

Se comparó los grados de adquisición de los estudiantes, en torno de los cuadriláteros, antes de la aplicación de la propuesta didáctica con los recogidos luego de la implementación de la propuesta, obteniéndose notables avances en los grados de adquisición de los niveles de razonamiento.

Por lo expuesto, se concluye que la propuesta didáctica de enseñanza de los cuadriláteros, basada en el modelo Van Hiele, con la ayuda del software GeoGebra, incrementó los grados de adquisición en torno del objeto matemático cuadriláteros.

La aplicación de la propuesta didáctica, conformada por las 19 actividades, las cuales siguieron las fases del Modelo Van Hiele, contribuyó y facilitó la comprensión de los estudiantes respecto al objeto matemático cuadriláteros.

Los estudiantes han mejorado su capacidad para justificar sus respuestas, la cual, en un inicio, estaba guiada por lo visual y supeditada por la utilización de figuras estereotipadas/estándar. Luego de la intervención, son poco frecuentes las veces que se dejan llevar por lo visual; por el contrario, empiezan a justificar sus respuesta usando algunas propiedades o, en todo caso, recurriendo a ejemplos y contraejemplos o ambos, para afirmar o negar un enunciado.

El software GeoGebra facilitó la comprensión de los cuadriláteros, ya que permitió manipular (realizar cambios en la figura de forma inmediata) e interactuar con el objeto cuadrilátero.

\section{REFERENCIAS BIBLIOGRÁFICAS}

1 Ministerio DE EdUCACión. Cómo rinden los estudiantes peruanos en Comunicación y Matemática: Resultados de la Evaluación Nacional 2001 Cuarto grado de secundaria informe pedagógico [Consultado: 17 de mayo de 2012]. Disponible en: http://www2.minedu.gob.pe/umc/ admin/images/documentos/archivo_4.pdf

2 Rizzolo S. Diseño de Actividades Geométricas Interactivas en el Marco Conceptual del Modelo de Van Hiele [Consultado el 03 de agosto de 2012]. Disponible en: http://www.coopvgg.com.ar/ sergiorizzolo/trabajo/trabajo_final.pdf

3 VAn Hiele P. El problema de la comprensión [tesis doctoral]. Utrecht: Universidad Real de Utrecht, Facultad de Matemática; 1957.

4 Corberán, R., Huerta, P., Margarit, J., PeÑas, P. \& Ruíz, E. Didáctica de la geometría: Modelo Van Hiele. España: Colecció: Educació. Materials; 1989.

5 GutiÉRREZ, A. \& JAIME, A. Aportaciones a la interpretación y aplicación del Modelo de Van Hiele: la enseñanza de las isomerías. La evaluación del nivel de razonamiento [tesis doctoral]. Valencia: Universidad de Valencia; 1993.

6 MAYBERRY, J. W. The van Hiele levels of geometric thought in undergraduate preservice teachers. Journal for Research in Mathematics Education; 1987, 14(1), 58-69. 